\section{Nucleic Acid-Based Microarrays and Nanostructures}

\section{INTRODUCTION}

$\mathbf{N}$ ucleic acid chemistry at the material science interface has rapidly gained importance as chemists develop new strategies for nucleic acid array-based detection systems. The fabrication of DNA microarrays requires attachment of both biologically and chemically derived nucleic acids to a variety of surfaces. Surface-attached nucleic acids can be modified in ways that will allow optical or electrical detection of hybridization, extension, or other nucleic acid-based transformations. The chemistries of nucleic acid surface attachment and modification are also related to efforts to develop nucleic acids as material for fabrication of nanostructures. Their highly specific self-assembling properties, coupled with their potential for synthetic remodeling, provide the opportunity to use nucleic acid-related substances as scaffolds as well as functioning components of mechanical, electronic, or photonic devices. This chapter will provide a means to highlight advances in the field and provide detailed protocols for some of the potentially more useful applications.

To initiate the chapter, one of the pioneers of the field, Nadrian Seeman, provides a perspective of the advances in his laboratory. In UNIT 12.1, "Key Experimental Approaches in DNA Nanotechnology," Dr. Seeman describes the construction of supramolecular structures based on DNA self-assembly. Important issues concerning synthesis strategy, junction design, and branching motifs are described. The characterization of complex DNA supramolecular structures is discussed in detail. This information provides important guidelines for future experimenters pursuing the construction of self-assembling supramolecular structures based on nucleic acids.

In UNIT 12.2, "Preparation of Gold Nanoparticle-DNA Conjugates," Andrew Taton provides experimental protocols for the preparation of gold nanaparticles containing a single tethered oligonucleotide, as well as gold nanoparticles coated with a dense layer of oligonucleotides. The unit includes protocols that provide specific guidelines for preparation of nanoparticles of different sizes, as well as their characterization and properties. Recent research on gold nanoparticle-linked oligonucleotides suggests that they may be of significant use as components of hybridization-based diagnostic probes.

UNIT 12.3, "Synthesis of 5'-Phosphoramidites Containing a Photolabile 3'-O-Protecting Group," is the first of a series of units on building oligonucleotides on surfaces. In UNIT 12.3, Markus Beier and Jörg Hoheisel outline procedures for the construction of deoxyribonucleoside $5^{\prime}$-phosphoramidites containing a photolabile protecting group on the $3^{\prime}$-hydroxyl group. These protected monomers allow one to build oligonucleotides in the $5^{\prime}$-to-3' direction, which yields surface-bound oligonucleotides with a $3^{\prime}$-terminus that is accessible for enzymatic extension. This oligonucleotide synthesis approach will be presented in an upcoming supplement (UNIT 12.5).

In the protocol that follows, UNIT 12.4, these authors describe procedures for derivatization of glass and polypropylene surfaces. The unique feature of the surface modification strategy described in this unit is the dendrimeric layer formed through a series of

Contributed by Donald E. Bergstrom

Current Protocols in Nucleic Acid Chemistry (2004) 12.0.1-12.0.2

Copyright $(C 2004$ by John Wiley \& Sons, Inc.
Nucleic

Acid-Based

Microarrays and

Nanostructures

12.0.1

Supplement 19 
coupling reactions between 4-nitrophenyl chloroformate or acryloyl chloride and polyamines. Dendrimeric layers terminating in an amino group may be used directly for in situ DNA synthesis or, after transformation to an acrylamide or 4-nitrophenylformate moiety, as a substrate for attachment of amino-labeled oligonucleotides.

Methods for linking oligonucleotides to glass surfaces for making arrays continue to improve. In UNIT 12.6, Melynk and coworkers outline procedures for making semicarbazide glass slides and alpha-oxo aldehyde conjugated oligonucleotides. The alpha-oxo aldehyde reacts rapidly with the semicarbazide to yield a highly stable alpha-oxo semicarbazone linkage. This results in arrays with superior sensitivity.

Donald E. Bergstrom 